\title{
Modelo de Apresentação Adaptativa de Objeto de Aprendizagem baseada em Estilos de Aprendizagem
}

\author{
Zenaide Carvalho da Silva ${ }^{1}$, Leandro Rodrigues Ferreira ${ }^{1}$, Andrey Ricardo \\ Pimentel $^{1}$ \\ ${ }^{1}$ Programa de Pós-Graduação em Informática - Universidade Federal do Paraná (UFPR) \\ Caixa Postal 19.081 - 81.531-980 - Curitiba - PR - Brasil \\ \{zcsilva, lrferreira, andrey\}@inf.ufpr.br
}

\begin{abstract}
Learning styles (LS) refer to preferences regarding ways and forms that the student prefers to learn. The knowledge of these styles allows propositions for teaching rearranging instructional methods and teaching strategies to enable more adapted to individual student learning needs. In this context, this paper presents an adaptive presentation model of learning object (LO) based on LS to provide adjustment in the presentation of LO according to the LS of the student.
\end{abstract}

Resumo. Os estilos de aprendizagem (EA) referem-se a preferências em relação aos modos e formas que o aluno prefere aprender. $O$ conhecimento desses estilos permite fazer proposições para o ensino rearranjando os métodos instrucionais e as estratégias de ensino para possibilitar uma aprendizagem mais adaptada as necessidades individuais do aluno. Neste contexto, este artigo apresenta um modelo de apresentação adaptativa do objeto de aprendizagem (OA) baseado em EA para proporcionar adaptação na apresentação do OA de acordo com o EA do aluno.

\section{Introdução}

Os estilos de aprendizagem (EA) referem-se as preferências individualizadas de uma pessoa, em relação aos modos e formas que ela prefere aprender no processo de ensino e aprendizagem.

Considerando que cada aluno tem sua maneira de receber e processar as informações, conhecer os EA é importante para compreender melhor suas preferências individuais, e entender porque certos métodos e técnicas de ensino funcionam melhor em alguns alunos, enquanto que em outros não funcionam. Os EA considerados como critério para adaptação de conteúdo em sistemas voltados para educação é um dos temas que estão cada vez mais presentes em pesquisa nacionais no âmbito educacional [Aguiar, Fechini e Costa 2014].

A apresentação adaptativa do objeto de aprendizagem (OA) considerando o EA do aluno é uma das possibilidades que permite oferecer aos alunos recursos educacionais digitais adaptados as suas preferências individuais de aprendizagem. Nesse caso esperase obter uma motivação maior ao aluno com o uso desse tipo de recurso educacional, pois o OA será apresentado de uma forma que respeita suas preferências individuais de aprendizagem. 
V Congresso Brasileiro de Informática na Educação (CBIE 2016)

Anais do XXVII Simpósio Brasileiro de Informática na Educação (SBIE 2016)

Existem diversos modelos de EA como o modelo de Kolb e o modelo de FelderSilverman [Felder and Silverman 1988, Honey and Munford 2000, Kolb 1984] que apresentam uma série de especificidades que precisam ser observadas quando escolhemos utilizar o EA como critério de adaptação para apresentação do material de aprendizagem, no caso o OA, de acordo com esses estilos.

Dentre esses modelos o modelo de Felder-Silverman (1988) foi elaborado com base em observações no ambiente escolar, sendo o mais adequado para instituições de ensino, mais consistente com as preferências dos alunos, sendo isso uma das razões pelas quais esta pesquisa utilizou os EA deste modelo para ser usado como critério de adaptação para criação do modelo de apresentação adaptativa do OA.

Nesta perspectiva, esse artigo apresenta um modelo de apresentação adaptativa do OA considerando os EA do modelo Felder-Silverman, no qual as preferências e características de cada estilo foram identificadas e mapeadas para "características de apresentação para $O A$ ", e formaram a base para definição dos critérios de adaptação, objetivando contribuir para criação de OA adaptados ao EA do aluno.

O texto esta estruturado da seguinte forma. A seção 2 apresenta uma revisão sobre EA. A seção 3 apresenta os trabalhos relacionados. A seção 4 descreve aspectos metodológicos. A seção 5 apresenta o modelo de apresentação adaptativa. A seção 6 discute aspectos do modelo sobre adaptação de material de aprendizagem; e, por fim, na Seção 7, são feitas as considerações finais e sugeridos trabalhos futuros.

\section{Estilos de Aprendizagem (EA)}

A definição mais comumente aceita é de Keefe (1997), considera o EA uma composição de características cognitivas, afetivas e fatores fisiológicos que servem como indicadores relativamente estáveis de como um aluno percebe, interage e responde ao ambiente de aprendizagem.

Existem vários modelos de EA, que foram elaborados por diversos autores, que podem ser usados pelos sistemas educacionais para representar os estilos dos alunos [Felder and Silverman 1988, Honey and Munford 2000, Kolb 1984]. Os estilos de aprendizagem são definidos por esses autores de forma distintas, influenciados por diferentes teorias da psicologia da aprendizagem.

Esta pesquisa utilizou o modelo de Felder-Silverman (1988), por ser considerado o mais indicado para ser utilizado em ambientes voltados para a educação, e adequação melhor de suas escalas às características dos materiais de aprendizagem, e também mais utilizado no contexto brasileiro e internacional em pesquisas relativas à adaptação e personalização de materiais de aprendizagem, além de prover um bom grau de adaptatividade aos perfis dos alunos [Aguiar, Fechini e Costa 2014, Carvalho et al 2014, Graf, Kinshuk e Liu 2009, Valaski, Malucelli e Reinerhr 2011].

O modelo de Felder-Silverman (1988) foi desenvolvido pelo professor Richard M. Felder e pela psicóloga Linda K. Silverman, que classifica os alunos em número de escala de acordo com a forma com que cada aluno recebe e processa as informações. $\mathrm{O}$ modelo proposto classifica o aluno em quatro dimensões: a) Percepção da Informação (Sensorial x Intuitivo); b) Retenção da informação (Visual x Verbal); c) Processamento da informação (Ativo x Reflexivo) e d) Organização da informação (Sequencial x 
V Congresso Brasileiro de Informática na Educação (CBIE 2016)

Anais do XXVII Simpósio Brasileiro de Informática na Educação (SBIE 2016)

Global). As características dos alunos de acordo com seu EA para cada dimensão são:

-Percepção da informação: aluno "sensorial" aprende fatos, resolve problemas com métodos estabelecidos, é mais detalhista, é metódico; enquanto que o "intuitivo" prefere descobrir possibilidades e relações, gosta de novidades e não gosta de repetição, prefere mais conceitos e teorias, tende a ser mais teórico, é mais rápido no trabalho e mais inovador.

-Retenção da informação: aluno "visual" recorda mais facilmente do que viu como figuras, fluxogramas, filmes e demonstrações, privilegia as informações que recebe através de imagens, diagramas, gráficos e esquemas; o "verbal" tem mais facilidade com as palavras, explicações escritas ou orais, privilegia o que é falado, o que está escrito, as fórmulas.

-Processamento da informação: aluno "ativo" compreende e retém melhor a informação trabalhando de modo ativo, agindo sobre algo, discutindo e aplicando conceitos ou explicando para os outros, gosta mais do trabalho em equipe, tende a ser mais experimentalista; o "reflexivo" prefere primeiro refletir sobre a informação recebida, gosta mais de trabalhar individualmente, tende a ser mais teórico.

-Organização da informação: aluno "sequencial" aprende de forma linear, em etapas sequenciadas, passo a passo, segue caminhos lógicos para encontrar soluções, apresenta capacidade de análise; o "global" aprende em grandes saltos, assimilando o material quase aleatoriamente, sem ver as conexões, para então, compreender o todo, hábil para resolver problemas complexos rapidamente, mas tem dificuldades para explicar como fez, apresenta capacidade de síntese.

Esse modelo utiliza como instrumento de mensuração o ILS (Index of Learning Styles) para identificar os EA baseados em FSLSM (Felder and Silverman learning Style Model), que compreende quarenta e quatro perguntas, onze para cada uma das quatro dimensões anteriormente descritas, mais detalhes em [Felder e Soloman 2006].

\section{Trabalhos Relacionados}

Silva e Dorça (2014) definiram uma nova abordagem para personalização automática do processo de ensino em sistemas adaptativos e inteligentes para educação à distância, levando em conta os EA dos estudantes. Criaram um método de adaptação que utiliza uma modelagem de preferências de aprendizagem representadas através de distribuições de probabilidades ao invés de tipos fixos, o que resultou em processos de modelagem do estudante e personalização do processo de ensino não-determinísticos, possibilitando o tratamento das incertezas inerentes ao processo de modelagem de EA e adaptação de conteúdo.

Yang, Hwang e Yang (2013) desenvolveram um sistema de aprendizagem adaptativa considerando várias dimensões de características personalizadas, propôs um módulo de apresentação personalizado para o desenvolvimento de sistemas de aprendizagem adaptativa com base no modelo de estilo cognitivo campo dependente/independente e os EA do modelo de estilo de aprendizagem de FelderSilverman. Seus resultados experimentais mostraram que a abordagem proposta é capaz de ajudar os alunos a melhorar o seu desempenho no processo de aprendizagem. 
V Congresso Brasileiro de Informática na Educação (CBIE 2016)

Anais do XXVII Simpósio Brasileiro de Informática na Educação (SBIE 2016)

O trabalho de Fasihuddin, Skinner e Athauda (2014) apresentou uma proposta de um modelo adaptável para personalizar os ambientes de aprendizagem abertos com base no modelo de EA de Felder-Silverman. Este modelo consiste de dois agentes principais para executar suas funcionalidades, o agente de identificação é responsável pela identificação dos estilos de aprendizagem dos alunos, monitorando certos padrões de comportamento dos alunos com os objetos de aprendizagem, enquanto o aluno interage com materiais de aprendizagem; e o agente de recomendação é responsável por fornecer um apoio de navegação adaptável com base nos EA e preferências identificadas.

Os trabalhos apresentados nessa seção utilizaram os EA para adaptar e/ou personalizar os ambientes de aprendizagem, ou adaptar a apresentação do material de aprendizagem, no entanto, não foram encontrados estudos aprofundados que explorem como utilizar as características de cada estilo dos modelos mapeados em relação a aspectos de interface e interação de OA para prover adaptação da apresentação de conteúdos dos materiais de aprendizagem.

\section{Metodologia}

Uma revisão da literatura foi realizada através da busca e investigação dos principais modelos de EA e de maior relevância, de acordo com alguns estudos pesquisados [Akbulut and Cardak 2012, Al-Azawer and Badii 2014, Cofield et al. 2004, Velusamy and Anouncias 2013]. Os modelos de EA estabelecem as formas de se classificar um aluno segundo suas preferências individuais de aprendizagem, através de seus estilos.

Nessas classificações definidas nos modelos são identificadas e estabelecidas características que são relevantes para serem consideradas no processo de adaptação de material de aprendizagem adequada ao estilo do aluno.

A partir dessa revisão foi escolhido o modelo de EA de Felder-Silverman (1988), utilizado como base teórica para ser estudado mais profundamente, o que possibilitou levantar e identificar preferências e características dos estilos desse modelo. Essas preferências e características foram mapeadas para "características de apresentação para $O A$ " em relação a sequenciamento, apresentação e forma/formato de conteúdo e recursos que compõem um OA.

Esse modelo foi escolhido por ser considerado o mais indicado para ser utilizado em ambientes voltados para a educação, e adequação melhor de suas escalas às características dos materiais de aprendizagem, e também mais utilizado no contexto brasileiro e internacional em pesquisas relativas à adaptação e personalização de materiais de aprendizagem, além de prover um bom grau de adaptatividade aos perfis dos alunos [Aguiar, Fechini e Costa 2014, Carvalho et al 2014, Graf, Kinshuk e Liu 2009, Valaski, Malucelli e Reinerhr 2011,].

A partir das "características de apresentação para OA" foi possível estabelecer os atributos mais relevantes que foram usados na criação de uma proposta de apresentação adaptativa do OA que considera como critério de adaptação o EA.

\section{Apresentação Adaptativa baseada em Estilos de Aprendizagem}

$\mathrm{Na}$ definição do modelo de apresentação adaptativa do OA foram consideradas as características e preferências dos EA do modelo de Felder-Silverman (1988), mapeadas 
V Congresso Brasileiro de Informática na Educação (CBIE 2016)

Anais do XXVII Simpósio Brasileiro de Informática na Educação (SBIE 2016)

para "características de apresentação para $O A$ " em relação a sequenciamento, apresentação e forma/formato de conteúdo e recursos que compõem um OA, adequados a cada estilo que estabeleceu a definição da estrutura da apresentação do OA para que ele fique adaptado ao estilo do aluno.

Essa estrutura foi projetada e composta respeitando os princípios da Teoria Cognitiva da Aprendizagem Multimídia (TCAM) de Mayer (2005), pois os princípios dessa teoria ajudam a evitar o uso inadequado de recursos nos mais variados formatos, que podem acarretar na distração e desmotivação do aluno no uso desse tipo de recurso, podendo causar insucesso no processo de aprendizagem.

A partir do estudo e investigação aprofundada sobre as "características de apresentação para $O A$ " em relação a sequenciamento, apresentação e forma/formato de conteúdo e recursos que compõem o OA, levantadas a partir de uma análise aprofundada das propriedades dos estilos do modelo de Felder-Silverman (1988), foi possível estabelecer os parâmetros e atributos necessários para definição do modelo.

Esses parâmetros e atributos foram definidos levando em consideração os elementos que podem ser alterados e/ou adaptados na criação e composição do OA para adaptar-se de acordo com cada EA.

$\mathrm{Na}$ definição da estrutura do modelo consideramos que na criação do OA ele será formado por "elementos de composição de conteúdo" que constituem as etapas: Resumo (Res): que deve fornecer uma visão geral e clara do conteúdo que será abordado; Introdução (Int): composto por um conteúdo breve para apresentação do assunto a ser estudado de um domínio; Desenvolvimento (Des): composto por um conteúdo mais abrangente que contempla o assunto de um domínio de uma forma mais completa; Atividade (Ati): formado por um conteúdo para fixação do assunto; e, Avaliação $(A v a)$ : avaliação do conteúdo abordado de um domínio. Esses "elementos de composição de conteúdo" são organizados em relação aos parâmetros e atributos definidos no modelo de apresentação adaptativa do OA, a seguir uma descrição desses parâmetros e atributos:

- Recurso $(R)$ : define quais os tipos de recursos que podem ser utilizados nos elementos da composição de conteúdos para apresentação do OA. Os recursos atribuídos no modelo podem ser: Video ( Vid); ou Diagrama (Dia); ou Gráfico (Gra); ou Figura (Fig); ou Narração (Nar); ou Palestra (Pal); ou Slide (Sli); ou Auto-Avaliação (Aav); ou Tabela (Tab); ou Experimento (Exp); ou Exercício (Exe); ou Simulação (Sim); ou Questionário (Que); ou Esquema (Esq); ou Animação (Ani); ou Foto (Fot); ou PáginaWeb (Pag); ou Mapa (Map); ou Demonstração (Dem); ou Exemplo (Exem).

- Forma de Exploração $(F E)$ : define como o conteúdo pode ser estruturado em relação a forma de exploração do conteúdo pelo aluno. Pode ser em Rede $(R e d)$ - investigação mais aleatória, sem seguir um roteiro; ou Linear ( $\operatorname{Lin})$ - investigação mais dirigida, com roteiro a seguir.

- Ordem de Detalhamento $(O D)$ : estabelece como o aluno prefere abordar os conteúdos apresentados no OA. Pode ser Específico-para-Geral (Esp-p-Ger): inicia na parte específica e prossegue para a parte geral para compreensão de um todo; ou Geral-paraEspecífico (Ger-p-Esp): inicia na parte geral e prossegue para a parte específica para compreensão de um todo. 
V Congresso Brasileiro de Informática na Educação (CBIE 2016)

Anais do XXVII Simpósio Brasileiro de Informática na Educação (SBIE 2016)

- Ordem de Composição $(O C)$ : define a organização das etapas usadas na composição dos conteúdos de um OA, ou seja, a ordem em que essas etapas serão apresentadas para o aluno. São três ordens de composição definidas: ordem $1-1^{\circ}$ Introdução, $2^{\circ}$ Desenvolvimento, $3^{\circ}$ Resumo, $4^{\circ}$ Atividade, $5^{\circ}$ Avaliação; ordem 2 - $1^{\circ}$ Introdução, $2^{\circ}$ Desenvolvimento, $3^{\circ}$ Atividade, $4^{\circ}$ Resumo, $5^{\circ}$ Avaliação; e ordem $3-1^{\circ}$ Resumo, $2^{\circ}$ Introdução, $3^{\circ}$ Desenvolvimento, $4^{\circ}$ Atividade, $5^{\circ}$ Avaliação.

Uma forma simplificada da composição da apresentação adaptativa da interface do OA pode ser representada na formulação abaixo:

$$
\text { InterfaceEstilo }(E)=\sum\left(\mathrm{OC}(x), \mathrm{OD}(j), \mathrm{FE}(k), \mathrm{R}\left(r_{1}, r_{2}, \ldots, r_{n}\right)\right)
$$

Onde: $E$ indica os estilos do modelo de Felder-Silverman, descritos na secção 3; $x$ pode assumir 1,2 e 3, que indica respectivamente, primeira, segunda e terceira ordem de composição; $j$ pode assumir 1 = "específico-para-geral" e 2 = "geral-para-específico"; $k$ pode assumir $1=$ "rede" e $2=$ "linear"; $r_{i}$ são os recursos que podem ser usados na composição do $\mathrm{OA}$; $O C$ indica a ordem de composição que as etapas usadas na composição dos conteúdos serão apresentadas no $\mathrm{OA}$; $O D$ indica a ordem de detalhamento de cada etapa do $\mathrm{OA} ; F E$ indica a forma de exploração que será usada na apresentação do $\mathrm{OA} ; R$ indica os recursos que podem ser usados na composição do OA.

Para cada estilo do modelo de Felder-Silverman (1988) foram criadas regras de adaptação para composição da interface do OA de acordo com esses estilos. A tabela 1 apresenta os atributos e parâmetros do modelo preenchidos com os valores referentes a cada estilo.

Tabela 1. Análise dos estilos do modelo de Felder-Silverman (1988) em relação aos atributos e parâmetros do modelo proposto.

\begin{tabular}{|c|c|c|c|c|}
\hline Estilo & Recurso (R) & $\begin{array}{l}\text { Forma de } \\
\text { Exploração } \\
\text { (FE) }\end{array}$ & $\begin{array}{c}\text { Ordem de } \\
\text { Detalhamento (OD) }\end{array}$ & $\begin{array}{c}\text { Ordem de } \\
\text { Composição } \\
\text { (OC) }\end{array}$ \\
\hline Ativo & $\begin{array}{l}\text { vid, dia, gra, fig, } \\
\text { aav, exe, sim, esq, } \\
\text { pag, map, exem. }\end{array}$ & rede & geral-para-específico & ordem 2 \\
\hline Reflexivo & $\begin{array}{l}\text { dia, gra, pal, sli, aav, } \\
\text { tab, exe, sim, esq,ani, } \\
\text { pag, dem, exem. }\end{array}$ & linear & especifico-para-geral & ordem 1 \\
\hline Visual & $\begin{array}{l}\text { vid, dia, gra, fig, } \\
\text { sli,aav, tab, exp, exe, } \\
\text { sim, ani, fot, pag, map, } \\
\text { dem, exem }\end{array}$ & rede & geral-para-específico & ordem 3 \\
\hline Verbal & $\begin{array}{l}\text { dia, nar, pal, sli, aav, } \\
\text { tab, exe, que, pag, } \\
\text { dem, exem. }\end{array}$ & linear & especifico-para-geral & ordem 3 \\
\hline Global & $\begin{array}{l}\text { dia, gra, fig, sli, aav, } \\
\text { exp, exe, esq, pag, } \\
\text { map, exem. }\end{array}$ & rede & geral-para-especifico & ordem 3 \\
\hline Sequencial & dia, gra, fig, nar, pal, & linear & especifico-para-geral & ordem 2 \\
\hline
\end{tabular}


V Congresso Brasileiro de Informática na Educação (CBIE 2016)

Anais do XXVII Simpósio Brasileiro de Informática na Educação (SBIE 2016)

\begin{tabular}{|c|c|c|c|c|}
\hline & $\begin{array}{l}\text { sli,aav, exe, sim, que, } \\
\text { esq, ani, pag, dem, } \\
\text { exem. }\end{array}$ & & & \\
\hline Sensorial & $\begin{array}{l}\text { vid, gra, nar, sli, aav, } \\
\text { tab, exp, exe, que, pag, } \\
\text { map, dem, exem. }\end{array}$ & rede & especifico-para-geral & ordem 3 \\
\hline Intuitivo & $\begin{array}{l}\text { gra, fig, nar, pal, sli, } \\
\text { aav, exe, sim, que, ani, } \\
\text { fot, exem. }\end{array}$ & linear & geral-para-especifico & ordem 1 \\
\hline
\end{tabular}

Para composição do OA adaptado a cada estilo foram criadas as regras de adaptação seguindo os atributos e parâmetros definidos no modelo. Para cada estilo a apresentação adaptativa se modifica seguindo os atributos e parâmetros apresentados na tabela 1. Uma interface para simulação da apresentação adaptativa do OA foi definida e criada a fim de realizar uma validação inicial da proposta, e será apresentada na próxima seção.

\section{Resultados e Discussão}

Com o intuito de realizar uma validação inicial da proposta foi definido e implementado um protótipo de interface para analisar preliminarmente o modelo de apresentação adaptativa definido. Na implementação da interface foi criada uma base de regras de estilos que possui uma regra para cada estilo definido no modelo em questão. As ações em cada regra consistem em efetuar o preenchimento das informações de cada parâmetro do estilo selecionado. Primeiramente será feita a alocação da $O C$, depois a alocação dos $R s$, em seguida a $F E$ e por fim a $O D$. Estas ações obedecem à modelagem proposta para este preenchimento, de acordo com as regras de adaptação apropriada a cada estilo, no exemplo abaixo, a regra para o estilo ativo:

Nome da Regra = "estiloAtivo"

Condições:

$$
\text { Estilo = "Ativo" }
$$

Ações:

a) Crie uma $\underline{O C}$ na seguinte ordem: "Introdução", "Desenvolvimento", "Atividade", "Resumo" e "Avaliação",b) Disponibilize os seguintes $\underline{R s}$ :

"Vídeo", "Diagrama”, "Figura”, "Gráfico”, "Auto-avaliação”,

"Exercício", "Simulação", "Esquema”, "Página-Web”, "Mapa” e

"Exemplo";c) Indique a $\underline{F E}$ "Rede”;d) E indique a $\underline{O D}$ "Geral para Específico".

Nesse caso conforme observado na figura 1 os elementos que compõem a interface estão dispostos seguindo as regras de adaptação que foram criadas para modificar os elementos de acordo com as características de cada estilo. O "Índice de Conteúdo", que consiste na exibição das etapas e suas respectivas subetapas dos "elementos de composição de conteúdo" numa disposição de uma árvore hierárquica, formando nodos para as etapas e subetapas, e segue conforme a OC pertencente ao estilo. Os itens do "Índice de Conteúdo" são liberados conforme a FE do estilo selecionado. Ou seja, se a $\mathrm{FE}$ for linear $(\mathrm{FE}=$ "linear"), o item subsequente à subetapa atual será liberado somente se a etapa atual for concluída, sendo indicado por um botão 
V Congresso Brasileiro de Informática na Educação (CBIE 2016)

Anais do XXVII Simpósio Brasileiro de Informática na Educação (SBIE 2016)

na tela, que ao ser clicado/selecionado informa ao sistema para liberar próxima etapa/subetapa, ou seja, uma exploração mais dirigida no OA. Caso a FE seja rede ( $\mathrm{FE}=$ "rede"), todos os itens que incluem as etapas e/ou subetapas, bem como os elementos da "Área de Controle de Navegação" são habilitados, assim uma exploração mais aleatória pode ser realizada no OA.

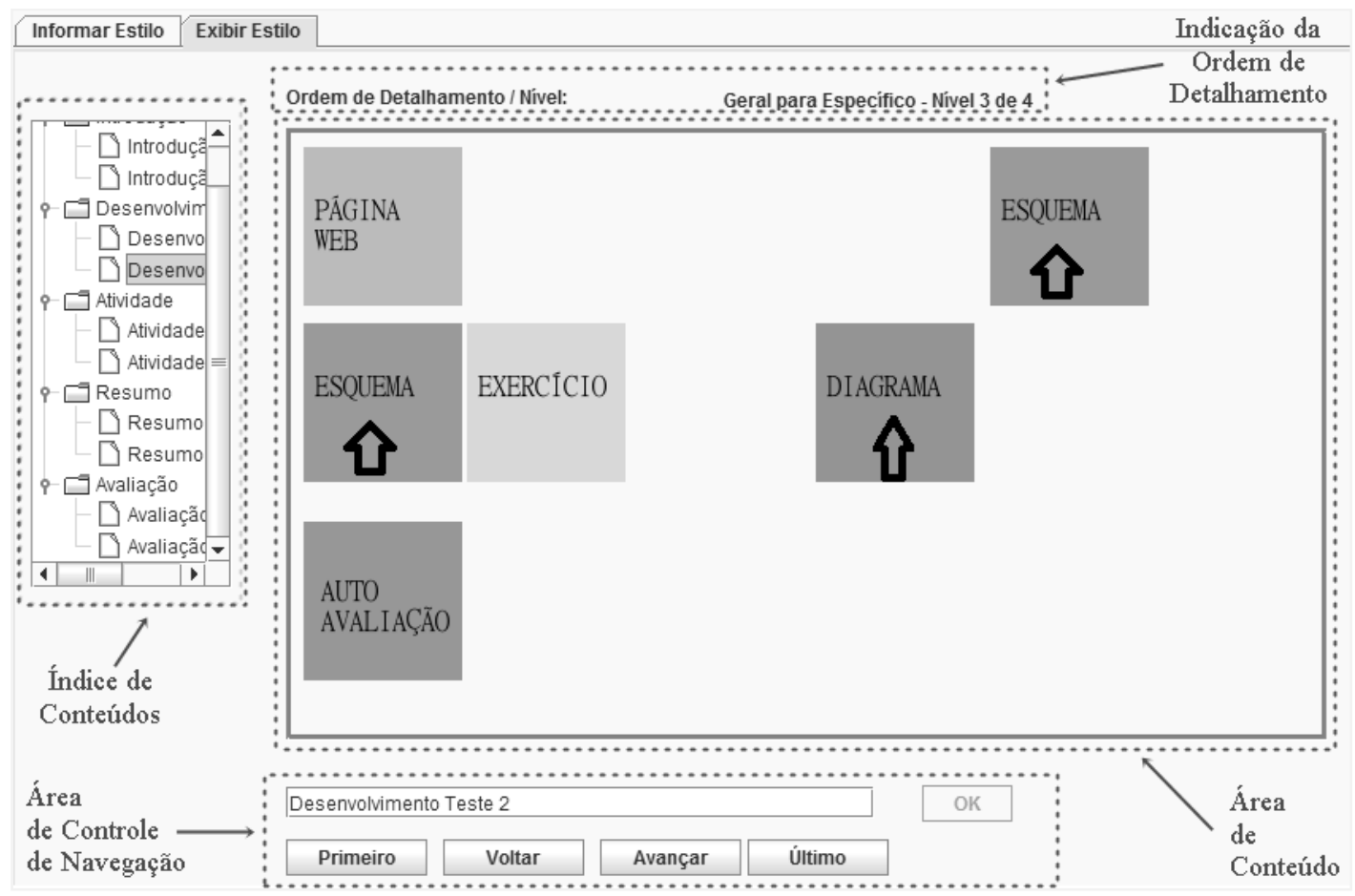

Figura 1. Tela da interface para o estilo ativo.

$\mathrm{Na}$ "Área de Conteúdo" são exibidos os recursos que compõem cada subetapa na criação do conteúdo do OA. Para garantir que os princípios da TCAM, como os princípios: multimídia (combinação de recursos no formato imagem e texto); proximidade espacial (quando um recurso no formato texto descrever um recurso no formato visual, estes devem ficar próximos), e o da modalidade (para todo o recurso animação deve-se usar o recurso narração ao invés de usar um texto escrito) sejam atendidos, foi estabelecido que no mínimo dois recursos sejam exibidos em cada subetapa na "Área de Conteúdo".

A "Indicação da Ordem de Detalhamento" está relacionada aos níveis que foram criados para demonstrar como o aluno prefere abordar os conteúdos apresentados pelo professor em um OA, respeitando a OD de cada estilo. Assim se a OD for "especificopara-geral" (OD="específico-para-geral"), o menor nível recebe o menor valor (no caso o valor "1") e o maior nível recebe o maior valor (no caso o valor da quantidade de níveis definida), caso a OD seja "geral-para-específico" (OD="geral-para-específico") acontece o inverso. Portanto foi adotado o menor valor para "mais específico" e o maior valor para o "mais geral".

A “Área Controle de Navegação" controla a exibição do "Índice de Conteúdo" e os botões de navegação, conforme a FE indicada pelo estilo informado e o fluxo de navegação entre as subetapas, seguindo a OC definida para o estilo. Conforme mencionado anteriormente a "Área Controle de Navegação" deve estar em conformidade com a FE definida para o estilo. Nesse caso se a FE for "rede" (FE = "rede"), todos os 
V Congresso Brasileiro de Informática na Educação (CBIE 2016)

Anais do XXVII Simpósio Brasileiro de Informática na Educação (SBIE 2016)

elementos da "Área Controle de Navegação" ficam habilitados permitindo ao aluno uma navegação de forma não sequencial ou aleatória nas etapas e subetapas compostas para o OA. Caso a FE seja "linear" (FE = "linear"), a navegação é sequencial, ou seja, passo a passo, o aluno precisa concluir a etapa/subetapa atual para prosseguir para a próxima etapa/subetapa.

Portanto para cada estilo do modelo de Felder-Silverman (1988) a interface sofreu mudanças para adaptar-se de acordo com os atributos e parâmetros do modelo de apresentação adaptativa definido, seguindo as regras de adaptação criadas para os estilos em relação a sequenciamento, apresentação e forma/formato de conteúdo e recursos que compõem um OA, propiciando OA adaptado e adequado ao EA dos alunos.

\section{Conclusões e Trabalhos Futuros}

Considerar os EA dos alunos na preparação e elaboração de material educacional possibilita atender as necessidades individuais em relação ao modo e forma que ele prefere aprender, contribuindo para um aprendizado mais adequado as suas preferências individuais de aprendizagem.

Esse trabalho trouxe contribuições para o processo de ensino e aprendizagem em relação a dois atores envolvidos: (a) o professor-autor, definiu e especificou um modelo de apresentação adaptativa do OA, que pode ser integrado na criação de ferramentas de autoria de fácil uso, com a diminuição da complexidade e esforço no momento da composição de OA adaptados ao EA do aluno, facilitando o trabalho do professor/autor; e (b) para o aluno, pois será beneficiado com OA adaptado ao seu EA, e consequentemente isso pode gerar um aumento da motivação em utilizar o OA pelo próprio aluno, já que o OA atenderá as suas preferências individuais de aprendizagem, e consequentemente poderá trazer melhorias no seu processo de aprendizagem.

Como trabalho futuro pretende-se implementar esse modelo como mecanismo de criação e composição de OA em ferramentas de autoria para criação de OA adaptados ao EA do aluno para conduzir experimentos no processo de ensino e aprendizagem.

\section{Agradecimentos}

Os autores agradecem a Coordenação de Aperfeiçoamento de Pessoal de Nível Superior (CAPES) pelo suporte parcial a esta pesquisa.

\section{Referências}

Aguiar, J. J. B.; Fechine, J. M.; Costa, E. (2014). Estilos Cognitivos e Estilos de Aprendizagem em Informática na Educação: um mapeamento sistemático focado no SBIE, WIE e RBIE. In: Anais do XXV Simpósio Brasileiro de Informática na Educação (SBIE), Dourados, p. 441-450.

Akbulut, Y. and Cardak, C. S. (2012). Adaptive Educational Hypermedia Accommodating Learning Styles: A Content Analysis of Publications from 2000 to 2011. Computers \& Education, vol. 58, n. 2, p. 835-842.

Al-Azawei, A. and Badii, A. (2014). State of The Art of Learning Styles-Based Adaptive Educational Hypermedia Systems (LS-BAEHSs). International Journal of Computer Science and Information Technology, vol. 6, n. 3, p. 1-19. 
V Congresso Brasileiro de Informática na Educação (CBIE 2016)

Anais do XXVII Simpósio Brasileiro de Informática na Educação (SBIE 2016)

Carvalho, V. C.; Dorça, F. A.; Cattelan, R. G.; Araújo, R. D. (2014). Uma Abordagem para Recomendação Automática e Dinâmica de Objetos de Aprendizagem Baseada em Estilos de Aprendizagem. In: Anais do XXV Simpósio Brasileiro de Informática na Educação - SBIE 2014, Dourados, MS, p. 1188-1197.

Coffield, F., Moseley, D., Hall, E. and Ecclestone, K. (2004). Learning Styles and Pedagogy in Post-16 Learning: A Systematic and Critical Review.

Fasihuddin, H., Skinner, G. and Athauda, R. (2014). Towards an Adaptive Model to Personalise Open Learning Environments Using Learning Styles. In Information, Communication Technology and System (ICTS), IEEE, p. 183-188.

Felder, R. M and B. Soloman (2006). Index of Learning Style Questionnaire. North Carolina State University. Disponível em $<$ http://www4.ncsu.edu/unity/lockers/users/f/felder/public/ILSpage.html $>$. Acesso em 20 set. 2012, 22:40.

Felder, R. M. and Silverman, L. K. (1988). Learning and Teaching Styles in Engineering Education. Journal of Engineering Education, 78(7):674-681.

Graf, S., Kinshuk and Liu, T. C. (2009). Supporting Teachers in Identifying Students' Learning Styles in Learning Management Systems: an Automatic Student Modelling Approach. Journal of Educational Technology \& Society, vol. 12, n.4, p. 3-14.

Honey, P. and Mumford, A. (2000). The Learning Styles helper's guide. Maldenhead Berks: Peter Honey Publications.

Keefe, J.W. (1997). "Learning Style: An Overview" in NASSP's Student Learning Styles: Diagnosing and Prescribing Programs" (pp. 1-17), Reston, VA: NASS.

Kolb, D. (1984). Experiential learning: Experience as the Source of Learning and Development. Prentice-Hall Englewood Cliffs, NJ.

Mayer, R. E. (2005). Principles for Managing Essential Processing in Multimedia Learning: Segmenting, Pretraining, and Modality Principles. In: MAYER, R. E., 2005a, p. 169-182.

Silva, D. H.; Dorça, F. A. (2014). Uma Abordagem Automática para Personalização do Processo de Ensino Baseada em Estilos de Aprendizagem em Sistemas Adaptativos e Inteligentes para Educação a Distância. Revista Brasileira de Informática na Educação (RBIE), v. 22, n. 2, p.1-15.

Valaski, J., Malucelli, A. e Reinehr, S. (2011). Revisão dos Modelos de Estilos de Aprendizagem Aplicados à Adaptação e Personalização dos Materiais de Aprendizagem. In XXII Simpósio Brasileiro de Informática na Educação — SBIE, p. 844-847.

Velusamy, B. and Anouncias, M. (2013). A Narrative Review of Research on Learning Styles and Cognitive Strategies. Journal Of Theoretical \& Applied Information Technology, v. 52, n. 1, p. 23-29, Computers \& Applied Sciences Complete.

Yang, T.C., Hwang, G.J. and Yang, S. J.H. (2013). Development of an Adaptive Learning System with Multiple Perspectives Based on Students' Learning Styles and Cognitive Styles. Educational Technology \& Society, 16 (4), 185-200. 